[Vicino Oriente XX (2016), pp. 121-128]

\title{
BOLLETTINO DELLE ATTIVITÀ DEL MUSEO DEL VICINO ORIENTE, EGITTO E MEDITERRANEO DELLA SAPIENZA, ANNO 2016
}

\author{
Daria Montanari - Sapienza Università di Roma
}

A summary of the activities and exhibits organized by the Museum VOEM in year 2016 is offered in this paper. They included workshops and conferences for the wide public, and seminars and trainings for scholars and researchers. As regards outreach, the Museum participated into numerous academic initiatives to promote and encourage the knowledge of the ancient cultures of the Near East, Egypt and the Mediterranean and to the many activities carried out by the Polo Museale Sapienza.

Keywords: research activity; guided tour; educational workshop; seminars; training activities

Il Museo del Vicino Oriente, Egitto e Mediterraneo nel corso del suo secondo anno di apertura al pubblico è stato promotore di numerose attività e ha preso parte alle iniziative e agli eventi organizzati dal Polo Museale Sapienza.

Contemporaneamente si è svolta l'ordinaria attività di ricerca sulle collezioni che lo costituiscono, con la partecipazione di studiosi, studenti, tesisti, specializzandi e dottorandi.

Le attività svolte presso il Museo e dal suo staff sono basilarmente ascrivibili alle tre missioni del Museo, la divulgazione (§ 1.), la formazione, caratterizzata anche quest'anno dalla qualificante esperienza dell’Alternanza Scuola Lavoro (§ 2.), e la ricerca (§ 3.), nell'intento di proseguire il manifesto ${ }^{1}$ proposto nell'anno $2015^{2}$.

Per quanto attiene alla curatela delle Collezioni, queste sono state oggetto di campagne di schedatura, documentazione e restauro (§ 4.). Alcuni reperti sono stati concessi in prestito per delle esposizioni ${ }^{3}$, e si sono anche registrate delle nuove accessioni (§ 5.)

\section{DiVULGAZIONE E OUTREACH}

Il Museo ha sviluppato un programma di incontri destinato al pubblico 'non addetto ai lavori' organizzando un palinsesto di conferenze e visite guidate. Queste ultime sono state attagliate ai diversi tipi di pubblico, scuole inferiori e scuole superiori, amatoriale ed esperto, che si è appassionato al Museo.

Per gli studenti delle scuole inferiori sono stati progettati alcuni laboratori didattici, incentrati sui temi dell'alimentazione degli antichi egizi e sulle prime scritture, durante i quali sono stati introdotti alcuni temi fondamentali delle culture vicino-orientali, quali l'agricoltura e l'alfabeto, attraverso forme interattive di gioco-studio.

Numerose visite guidate e laboratori didattici per alcune classi di IV elementare, dunque, sono stati tenuti in primavera e in estate (fig. 1).

1 Montanari 2015.

2 L'anno si era si era concluso con una conferenza tenuta dal Prof. Alessandro Campus il 28 dicembre dal titolo "Cartagine e il problema del Tofet".

3 Tra ottobre 2015 e gennaio 2016 due tra i reperti più rilevanti della collezione egiziana, il Piatto di Hotepsekhemuy (E2106) e la Testa di Faraone (E549), sono stati esposti nella mostra "La forza delle rovine", presso il Museo Nazionale Romano Palazzo Altemps (Nigro 2015). 
Alcune visite guidate per gruppi di adulti sono state tenute nel corso dell'intero anno. Tra queste, il Museo ha avuto l’occasione di accogliere la visita della Professoressa Serena Maria Cecchini dell’Università di Bologna, già illustre membro di numerose missioni archeologiche rappresentate nel Museo, nonché scopritrice dell'avorio simbolo del MVOEM.

In occasione del primo anniversario dell'inaugurazione del Museo, nel marzo 2016, è stata organizzata l'esposizione 'Dalla torre alla grotta'. Presentazione in anteprima delle scoperte della Sapienza a Betlemme, incentrata sulla scoperta e sui ritrovamenti della necropoli di Khalet al-Jam'a ${ }^{4}$.

Nel mese di maggio, aderendo all'iniziativa promossa dal Polo Museale Sapienza 'Sabato al Museo', il MVOEM ha proposto, nei giorni di sabato 7, 14, 21 e 28, un calendario speciale di visite guidate tematiche (fig. 3) e laboratori didattici, "Le immagini e le prime scritture", destinato ai bambini tra i 6 e i 12 anni e focalizzato su gli alfabeti e le scritture del Vicino Oriente antico, dell'Egitto e dei Fenici, sulle tecniche di scrittura e sui relativi supporti, con una sezione dedicata alla riproduzione degli alfabeti (fig. 2).

Sabato 14 maggio è stata inaugurata l'esposizione intitolata Save Palmyra ${ }^{5}$, alla presenza del Presidente del Polo Museale della Sapienza, Prof.ssa Marina Righetti, e del Direttore, Prof. Giorgio Manzi, e con gli interventi del Prof. Marcello Barbanera, Direttore del Museo dell'Arte Classica, e del Prof. Paolo Matthiae, nonché della giornalista Cinzia Dal Maso e del Prof. Lorenzo Nigro, Direttore del Museo del Vicino Oriente, Egitto e Mediterraneo (fig. 4)

In occasione della Notte Europea dei Musei, sabato 21 maggio, è stato organizzato l'evento Building Up the Round Tower, all'interno del quale è stato offerto al pubblico del Museo il concerto per chitarra classica tenuto dal Maestro Giuseppe Molino, la conferenza del Prof. L. Nigro 'Building Up The Round Tower'. Da Gerico a Castel Sant'Angelo e la conferenza del Prof. A. Campus Scritture.

Inoltre, per le giornate di Porte Aperte alla Sapienza 2016 - XX Edizione, 12-14 luglio, sono state attivate alcune visite guidate tematiche e nel giorno di mercoledì 13 luglio è stato organizzato l'evento Dig in the Levant and the Mediterranean - Scavare nel Levante e nel Mediterraneo (Palestina, Giordania, Libano, Cipro, Malta, Sicilia, Sardegna), all'interno del quale dal Prof. Lorenzo Nigro è stata tenuta la conferenza Batrawy: scavare una capitale nel deserto giordano.

Le attività dell'autunno 2016 sono state inaugurate il 19 settembre dal Tour Erasmus Sapienza dedicato a tutti gli studenti stranieri e dalla Giornata Europea del Patrimonio \#GEP 2016, 24 settembre 2016. Questa giornata è stata dedicata al tema del Tempo, svolgendo laboratori didattici e visite guidate mirati, dal titolo Il tempo della vita: dalle prime città nel Vicino Oriente alle colonie fenicie nel Mediterraneo e Il tempo nell'Al di là: tombe e corredi nell'Antico Egitto.

Nei mesi di ottobre e novembre 2016 il Museo ha ricevuto le visite di delegazioni di università estere, rispettivamente il 25 ottobre la delegazione della Federazione Russa e il 24 novembre il rettore e la rappresentanza francese de Université Aix en Marseille.

4 Nigro et al. 2015.

5 Pomar 2016. 
Martedì 29 novembre negli ambienti del MVOEM è stato presentato il volume Terre e cementi. Architetture e spazi a firma del Prof. Ettore Janulardo.

All'interno dell'apertura serale e notturna del 10 dicembre, prevista in occasione dell'iniziativa promossa dai musei civici di Roma Musei in Musica, il MVOEM ha ospitato il concerto per violoncello di Simone Chiominto, J.S. Bach: Suite in do minore, ed ha scandito la serata con alcune visite guidate tematiche.

L'anno 2016 si è concluso con la conferenza del Prof. Lorenzo Nigro dal titolo Gerico nei secoli: dal Bronzo Tardo al Ferro. Jericho across the ages: from Late Bronze to Iron Age, tenutasi martedì 20 dicembre.

\section{FORMAZIONE}

I percorsi di formazione dell'anno 2016 proposti dal MVOEM sono stati destinati, da un lato, agli studenti delle scuole superiori grazie al progetto Alternanza Scuola Lavoro 2016 (§ 2.1.) e, dall'altro, agli studenti universitari all’ interno dei programmi curriculari (§ 2.2.).

\subsection{Alternanza Scuola Lavoro 2016}

Il Museo VOEM ha aderito al progetto Alternanza Scuola-Lavoro 2016 promosso dalla Sapienza Università di Roma (http://www.uniroma1.it/archivionotizie/alternanza-scuolalavoro), accogliendo le richieste di cinque licei romani, Liceo Classico Plauto, Liceo Classico Bertrand Russell, Liceo Classico Cornelio Tacito, Liceo Classico Francesco Vivona, Liceo Linguistico Federigo Enriques, per un totale di 36 studenti. Il Museo, nella persona del Prof. Lorenzo Nigro come tutor Sapienza e delle Dott.sse Daria Montanari ed Elisabetta Gallo come referenti interni Sapienza, ha pianificato un percorso formativo di 36 ore per studente, dal titolo I giovani detectives dell'archeologia. L'indagine archeologica 2.0. 'Libro Bianco' dei reperti archeologici dispersi ritrovati dagli studenti dei licei romani.

Il percorso è stato articolato in 10 incontri: martedì 31 maggio, primo incontro inaugurale mirato all'informazione del gruppo di studenti sulla storia del MVOEM e delle sue collezioni, sul processo che subisce il reperto nell'iter scavo-museo, con particolare riferimento alle tematiche di patrimonio culturale, bene archeologico e salvaguardia, conservazione e musealizzazione; sei incontri applicativi concentrati sulle ricerche tematiche previste per il gruppo, sulla collezione dei dati, raccolta delle immagini e sulla compilazione delle schede di salvaguardia, 7, 9, 14, 16, 21 e 23 giugno; due incontri dedicati all'apertura del Museo, all'attività di curatore e assistente di sala, alla simulazione di visita guidata, all'analisi e al dibattito sui temi di recupero del patrimonio culturale, $11 \mathrm{e}$ 18 giugno; martedì 28 giugno, incontro conclusivo dedicato alla presentazione da parte degli studenti delle scoperte effettuate, alle relazioni finali e alla presentazione del 'Libro Bianco' (fig. 5).

Il progetto ASL 2016 svolto presso il MVOEM ha dato la possibilità agli studenti dei licei romani che hanno partecipato di accrescere le capacità di lavorare in team e in relazione a nuove esperienze; di apprendere e approfondire i concetti di patrimonio culturale e bene archeologico; di comprendere i metodi e delle fasi dell'indagine archeologica dal ritrovamento alla pubblicazione, attraverso la schedatura e la ricerca; di implementare le capacità di ricerca online, in particolare dei reperti archeologici, e più in 
generale le capacità informatiche, alcune funzioni redazionali, tramite l'utilizzo di programmi del pacchetto informatico Office; di apprendere il linguaggio tecnico archeologico, approfondendo alcune nozioni fondamentali della storia e della cronologia del Vicino Oriente, Egitto e Mediterraneo; di accrescere le capacità di presentazione e divulgazione.

Il prodotto finale di tale progetto è il 'Libro Bianco', un libro che raccoglie i reperti selezionati e schedati dagli studenti dei licei, provenienti da scavi illegali, trafugati o frutto di traffici illeciti.

\subsection{Seminari universitari}

In ricordo della Professoressa Antonia Ciasca, che tanta della sua vita ha dedicato all'archeologia del Mediterraneo e alla quale il Museo deve parte delle sue collezioni, è stato istituito un ciclo di conferenze annuali. Tali conferenze hanno visto la partecipazione come oratori di studiosi illustri, che hanno avuto la fortuna di conoscere e lavorare con Antonia Ciasca, e di giovani ricercatori cha hanno iniziato il loro percorso proprio negli scavi di Mozia e che pongono i loro studi nel solco tracciato dalla Ciasca.

Le conferenze, dunque, sono state tenute dalla Dott.ssa Maria Pamela Toti (Museo G. Whitaker - Mozia), martedì 12 gennaio, con una presentazione dal titolo Nella cucina di Arishat. Una cuoca ci racconta l'alimentazione a Mozia; dal Dott. Adriano Orsingher (University of Oxford), giovedì 18 febbraio, con una presentazione dal titolo "Tante maschere e pochi volti”. Lungo le coste fenicie del Mediterraneo; dal Prof. Piero Bartoloni (Università di Sassari), giovedì 10 marzo ore 17, con un intervento dal titolo Il Tofet: un rito cruento o una pietosa necropoli infantile; dal Dott. Nicola Chiarenza (Rheinische Friedrich-Wilhelms-Universität Bonn), giovedì 7 aprile, con una presentazione dal titolo Persistenze e variazioni ai confini dell'eparchia cartaginese.

Nel giorno di giovedì 23 giugno il Museo ha ospitato la lecture della Prof.ssa Lillian Joyce (The University of Alabama in Huntsville) dal titolo Hadrian's cult statues: reconstructing Venus and Roma.

Due conferenze sono state tenute nel mese di novembre, rispettivamente il 22 e il 24: la Prof.ssa Marcella Frangipane (Sapienza) ha presentato Arslantepe: un potente centro politico al confine tra diversi mondi e civiltà, e la Prof.ssa Suzanne Richard (Gannon University) ha tenuto una lecture dal titolo Khirbat Iskander, Jordan: Early Bronze Age Urbanism, Collapse, and Renewal.

In apertura della conferenza della Prof.ssa Frangipane è stata presentata la guida del Museo, Compendio del Museo del Vicino Oriente, Egitto e Mediterraneo (ISBN 9788898154029), a cura del Direttore Lorenzo Nigro ${ }^{6}$.

\section{RICERCA}

Nel corso del 2016 sono stati avviati alcuni progetti di ricerca, studi su reperti e classi di manufatti conservati nel Museo. Tali studi si contraddistinguono per il carattere multidisciplinare, integrando indagini e analisi di tipo chimico, fisico e archeometrico, e nascono in seno a progetti pluriennali avviati dalle missioni archeologiche, relativi anche a

$6 \quad$ Nigro (a cura di) 2016. 
più siti. A questi si affiancano poi gli studi e le ricerche condotte all'interno delle attività didattiche curriculari, per le tesi di Laurea Magistrale, della Scuola di Specializzazione in Beni Archeologici e per i progetti del Dottorato di Ricerca.

\subsection{Lo studio delle prime produzioni ceramiche levantine}

Alcuni frammenti ceramici (VO3001/I-III; VO3021-3024, 3027-3028) provenienti dal sito di Gerico sono stati sottoposti ad analisi petrografiche, diffrazione a raggi X e scansione al microscopio elettronico per indagare i procedimenti di lavorazione delle prime ceramiche e le caratteristiche delle prime produzioni gerichiote, tra il periodo neolitico e il Bronzo Antico, all'incirca dal 6000 a.C. al 2500 a.C. Questo studio si inquadra in un più ampio progetto di ricerca dedicato all'analisi e alla definizione delle produzioni delle prime ceramiche e dei loro impasti nel Levante ${ }^{7}$.

\subsection{Lo studio della ceramica Protocorinzia di Mozia}

Un riesame sistematico della ceramica protocorinzia rinvenuta nel Tofet di Mozia durante gli scavi di Antonia Ciasca conservata presso il Museo è stato intrapreso nel secondo semestre del 2016. Questo studio si inquadra nel più ampio orizzonte della cronologia dell'espansione fenicia nel Mediterraneo, uno dei temi centrali del pluriennale progetto di ricerca Motya Living Lab, e rappresenta l'apparato catalogico di una tesi di laurea triennale dal titolo La ceramica protocorinzia dal Tofet di Mozia: scavi Antonia Ciasca 1964-1973.

\section{CuRATEla DELLE COLLEZIONI}

Nel corso dell'anno 2016 è stato impostato un programma volto alla documentazione e catalogazione dell'intera collezione del Museo.

Si è provveduto, infatti, primariamente alla stesura dell'inventario topografico completo dei reperti, in esposizione, conservati nei cassetti al di sotto delle vetrine e nel magazzino, attribuendo un numero di inventario identificativo a tutti i reperti che ancora non lo avessero ricevuto. Secondariamente, è stata strutturata una scheda tipo per la documentazione dei reperti, contenente i dati 'anagrafici', su provenienza e dimensioni, classificatori, riferimenti bibliografici, disegni e foto. Sulla base della scheda tipo è stato realizzato un database, caricato sul sito internet del Museo. Nel medesimo sito è stato predisposto un sistema di registrazione dei reperti/record di ricerca (query) impostato sulle voci principali del database, in modo tale da creare una piattaforma open access e facilmente consultabile contenente le collezioni del MVOEM ${ }^{8}$.

Grazie a questa attività di documentazione è stato effettuato un censimento completo dei reperti e del loro stato di conservazione, attuando interventi di pulizia, in alcuni casi, e restauro, in altri.

7 Medeghini et al. 2016. Lo studio è svolto in collaborazione con la Facoltà di Scienze Matematiche, Fisiche e Naturali della Sapienza, nella fattispecie con il Corso di Laurea in Tecnologia per la Conservazione e il Restauro dei Beni Culturali, Professoressa Caterina De Vito.

8 Il database è attualmente in corso di compilazione. 


\section{NUOVE ACCESSIONI}

Grazie alla collaborazione con la Rome La Sapienza Expedition to Palestine \& Transjordan (ROSEPAJ), missione archeologica attiva nel 2016 nei siti di Tell esSultan/Gerico, Tell Sheikh Abu Zarad e Betlemme, in Palestina, e nel sito di Khirbet alBatrawy, in Giordania, sono state registrate nuove accessioni nella Collezione Vicino Oriente del Museo. I reperti in questione sono rappresentati da vasellame ceramico, oggetti in pietra e ceramica, e lame di selce, d'età neolitica, del Bronzo Antico e Medio per il sito di Gerico, del Bronzo Medio e dell’Età del Ferro per Abu Zarad, del Bronzo Antico IV e Medio per Betlemme, del Bronzo Antico per il sito di Batrawy. L'acquisizione di tali materiali si inquadra all'interno dei progetti di ricerca, quale lo studio delle prime produzioni ceramiche levantine, e di alcuni Dottorati di Ricerca in Archeologia.

Nel corso dell'anno 2016 il Museo è stato aperto, grazie al contributo volontario degli studenti che partecipano alle attività scientifiche e seminariali.

\section{BIBLIOGRAFIA}

Medeghini, L. - Fabrizi, L. - De Vito, C. - Mignardi, S. - Nigro, L. - Gallo, E. - Fiaccavento, C. 2016 The ceramic of the "Palace of the Copper Axes" (Khirbet al-Batrawy, Jordan): A palatial special production: Ceramics International 42 (2016), pp. 5952-5962. MONTANARI, D.

2015 Bollettino delle attività del Museo del Vicino Oriente, Egitto e Mediterraneo della NigRO, L. Sapienza, anno 2015: Vicino Oriente XIX (2015), pp. 345-348.

2015 Memoria conservata, memoria violata: M. BARBANERA - A. CAPODIFERRO (a cura di), La NigRO, L. (a cura di) forza delle rovine, Catalogo della Mostra, Milano 2015, pp. 168-177.

2016 Compendio del Museo del Vicino Oriente, Egitto e Mediterraneo, Roma 2016.

Nigro, L. - MonTANARI, D. - Ghayyada, M. - YAsine, J.

2015 Khalet al-Jam'a. A Middle Bronze and Iron Age necropolis near Bethlehem (Palestine): Vicino Oriente XIX (2015), pp. 185-218.

POMAR, E.

2016 Save Palmyra. La distruzione del patrimonio archeologico nel Vicino Oriente. Perché e come ricostruire: Vicino Oriente XX (2016), pp. 111-120. 
XX (2016) Bollettino delle attività del Museo del Vicino Oriente, Egitto e Mediterraneo, anno 2016



Fig. 1 - Le visite guidate per le scuole elementari.

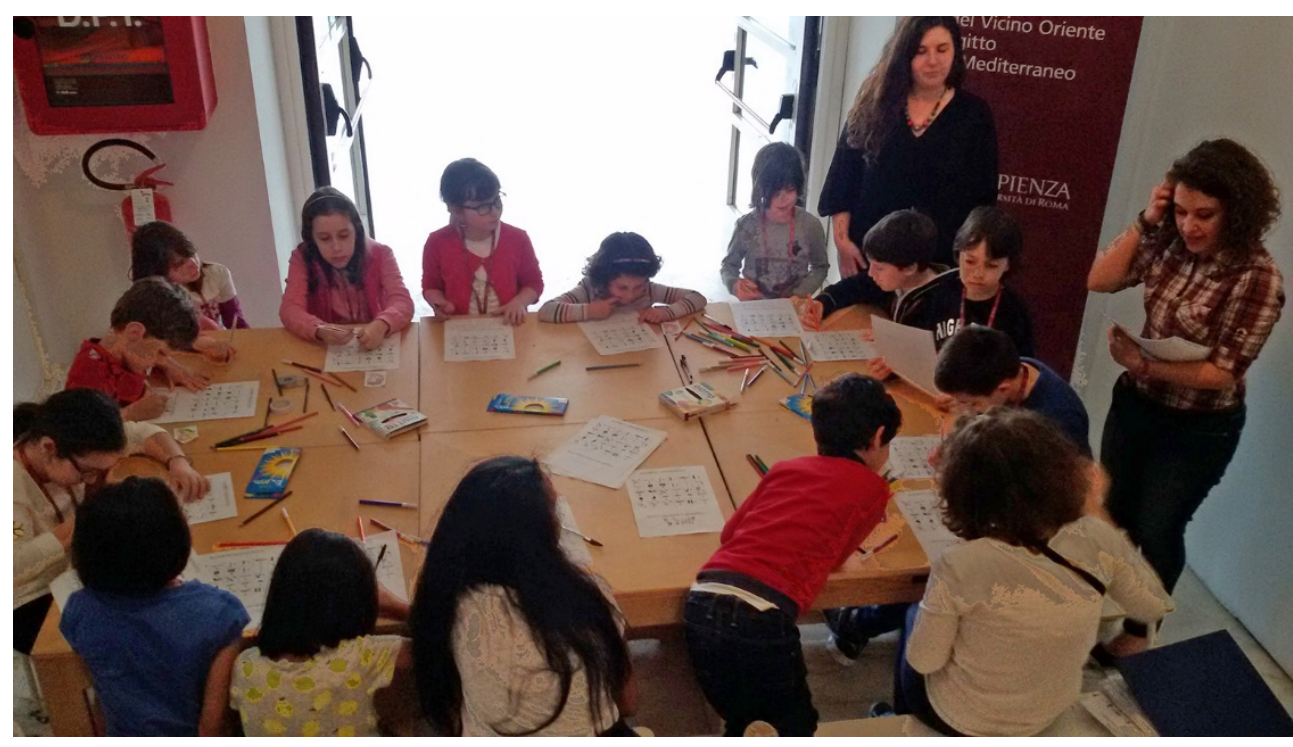

Fig. 2 - Il laboratorio didattico per i ragazzi tra 6 e 12 anni. 


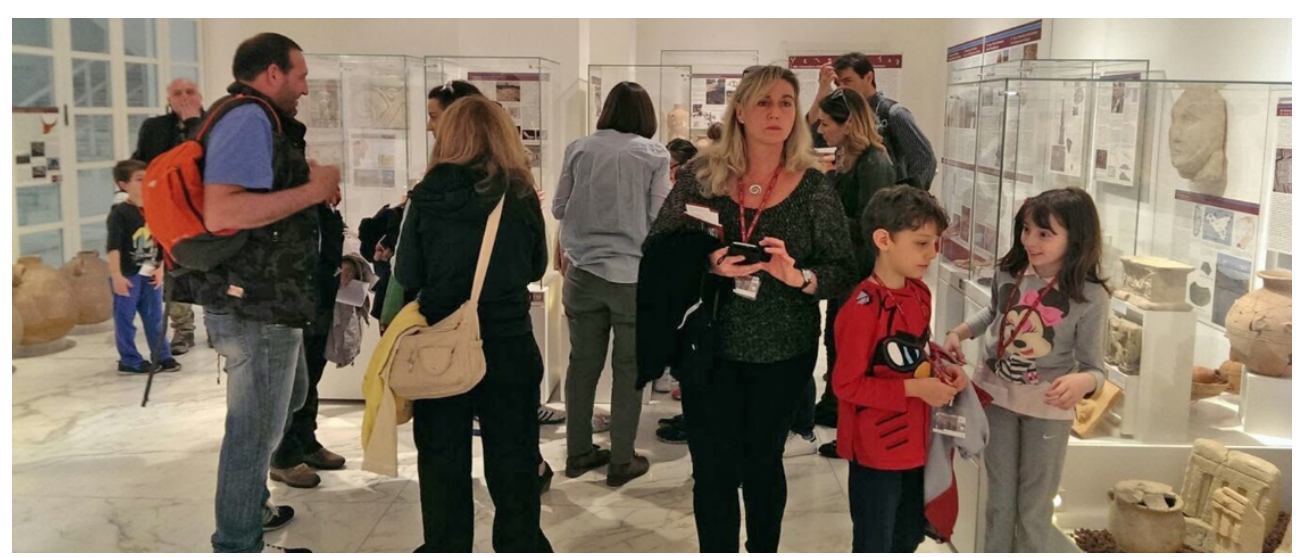

Fig. 3 - Il pubblico del Museo di ‘Sabato al Museo’.

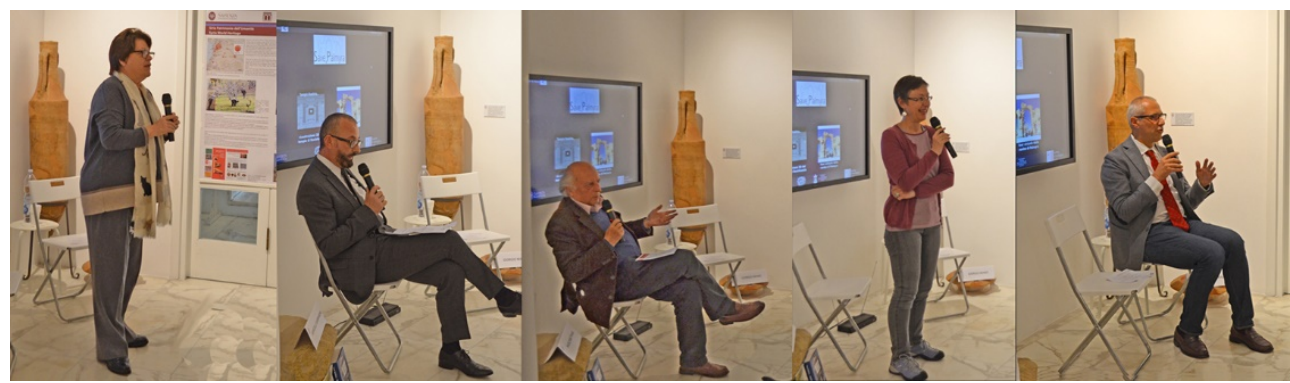

Fig. 4 - Alcuni momenti della presentazione della mostra Save Palmyra, 14 maggio 2016.

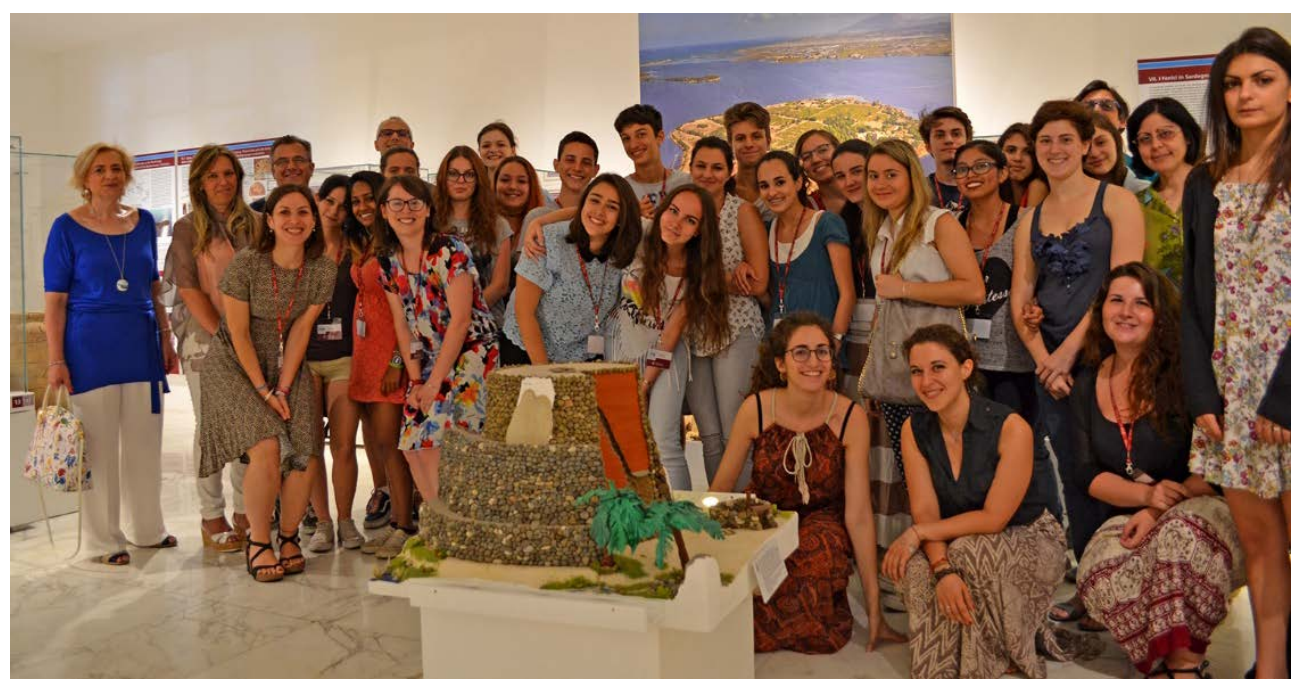

Fig. 5 - Foto di gruppo con i partecipanti alle attività ASL 2016 in occasione dell'evento conclusivo del 28 giugno 2016. 